\title{
Does psychache mediate the relationship between general distress and suicide ideation?
}

\author{
Rui C. Campos ${ }^{\mathrm{a}}$, Margarida Gomes ${ }^{\mathrm{a}}$, Ronald R. Holden ${ }^{\mathrm{b}}$, Margarida Piteirac, and Ana Rainha ${ }^{\mathrm{c}}$
}

a'Departamento de Psicologia, Escola de Ciências Sociais e Centro de Investigação em Educação e Psicologia, University of Évora, Évora, Portugal; 'Department of Psychology, Queen's University, Kingston, Ontario, Canada; 'Departamento de Psicologia, Escola de Ciências Sociais, University of Évora, Évora, Portugal

\begin{abstract}
This study evaluated whether psychache (i.e., mental pain) mediates the association between general distress, assessed as the frequency and the intensity of psychological symptoms in the previous week, and suicide ideation in community adults. For a sample of 202 adults, psychache fully mediated the relationship between suicide ideation and the frequency of psychological symptoms, and partially mediated the relationship between suicide ideation and the intensity of psychological symptoms. As such, mental pain fully or partially explains the process linking the frequency and the intensity of general distress to suicide ideation and, thus, mental pain is a target for potential intervention.
\end{abstract}

Suicide ideation is an important risk factor for suicidal behavior. Ideation has been linked to suicide attempts (Brezo et al., 2007) and to subsequent death by suicide. For example, in a 20 -year prospective study of almost 7,000 individuals, suicide ideation predicted subsequent death by suicide independently of previous suicide attempts, depressive disorders, psychiatric hospitalization, employment status, and age (Brown, Beck, Steer, \& Grisham, 2000).

Several factors have been empirically linked to suicidality, namely, clinical (e.g., depression; Thomson, 2012), demographic (e.g., male sex; Mościcki, 1997), and psychological variables (e.g., mental pain or psychache; Troister, D’Agata, \& Holden, 2015). Several models to explain suicide exist, namely psychosocial models of suicide that demonstrate the potential interactions between individual and social factors in different life stages (e.g., Beautrais, Collings, Ehrhardt, \& Henare, 2005).

Distress is related to suicide and suicide ideation. In fact, the majority of people who die by suicide previously presented with significant distress (Overholser, Braden, \& Dieter, 2012). For example, in a study of 485 participants in the community (Lund, Nadorff, \& Seader, 2015), individuals who had a psychiatric disorder were also those with the highest risk of suicide, even when controlling for depressive symptoms. However, it is also important to note that factors such as distress may contribute to suicide risk in some individuals but not in others, and that such factors may be relevant only when they occur in combination with particular stressors (Jacobs et al., 2010).

Among psychological variables related to suicidality, psychache (i.e., mental pain) is an important variable. Psychache is an affective phenomenon that has been clearly distinguished theoretically and empirically from general distress, depression, and hopelessness. Theoretically, Shneidman (1993) asserted the pre-eminence of psychache in suicide, arguing that psychache mediates the associations between all other psychological variables (e.g., general distress) and suicide. Whereas general distress is a nonspecific construct including symptoms (e.g., sadness, anxiety) of psychiatric disorders, psychache (i.e., mental pain) is a contiguous, but distinct, construct that is a continuing state of inner emotional turbulence or mental perturbation (Erinfolami et al., 2016; Shneidman, 2005). Psychache is a mental pain that is deeper, more primal, and more savage than general distress or depression, although general distress or depression may also be present (Erinfolami et al., 2016).

Empirically, research has shown associations between measures of psychache and suicidality with psychache having an added value beyond depression and hopelessness (DeLisle \& Holden, 2009; Mills, Green, \& Reddon, 2005; Troister \& Holden, 2010, 2013). Some studies have proposed a mechanism for this link (e.g., Campos et al., 2016), demonstrating that psychache mediates the
CONTACT Rui C. Campos rcampos@uevora.pt Departamento de Psicologia, Universidade de Évora, Apartado 94, Évora 7002-554, Portugal. 